\title{
BLer: A Boundary Labeller for Technical Drawings ${ }^{\star}$
}

\author{
Michael A. Bekos and Antonios Symvonis \\ National Technical University of Athens, \\ School of Applied Mathematical \& Physical Sciences, \\ 15780 Zografou, Athens, Greece \\ \{mikebekos, symvonis\}@math.ntua.gr
}

\begin{abstract}
BLer is a prototype tool aiming to automate the boundary labelling process [1. It targets the area of technical and medical drawings, where it is often common to explain certain features of the drawing by blocks of text that are arranged on its boundary.
\end{abstract}

\section{Introduction}

In technical drawings and medical atlases, it is quite common to place the labels on the boundary of the drawing and to connect them to the features they describe by non-crossing poly-lines (leaders). To the best of our knowledge, no drawing software includes support for automated placement of labels on (or near) the boundary of the drawing. BLer is a prototype tool that supports the boundary labelling model and facilitates the annotation of drawings with text labels. It is suitable for the production of medical atlases and technical drawings, where the basic requirement is large labels. It is entirely written in Java based on the yFiles class library (http://www.yworks.com).

\section{The Labelling Process}

BLer enables the user to quickly generate boundary labellings from scratch. Its environment (see Figure 1) supports multiple views of the labelling and directs the user through the steps of the labelling process (diagram loading, definition of enclosing rectangle, definition of point features, production of boundary labelling).

The labellings produced by the current version are based on algorithms developed by Bekos et. al. 11. They minimize the total leader length and contain no crossings. The resulting drawings are simple, in terms of readability, ambiguity and legibility.

BLer supports several labelling models. These include: the sides of the enclosing rectangle where labels can be placed, the type of the leaders (opo, po, or $s$ [1]), the type of the ports.

\footnotetext{
* This work has been partially founded by the program "Pythagoras" which is cofunded by the European Social Fund (75\%) and Greek National Resources (25\%).
} 


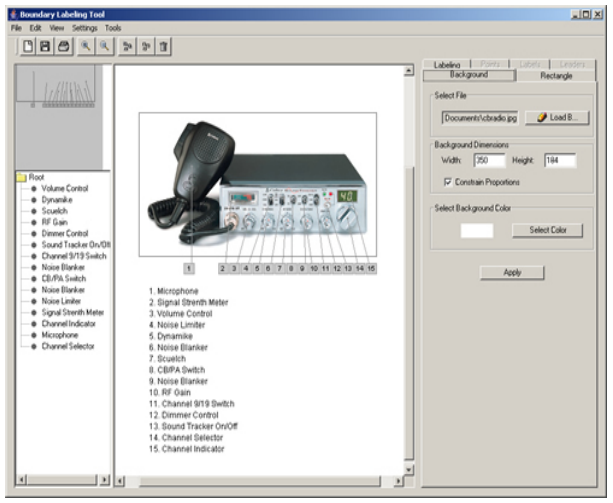

Fig. 1. A snapshot of Bler

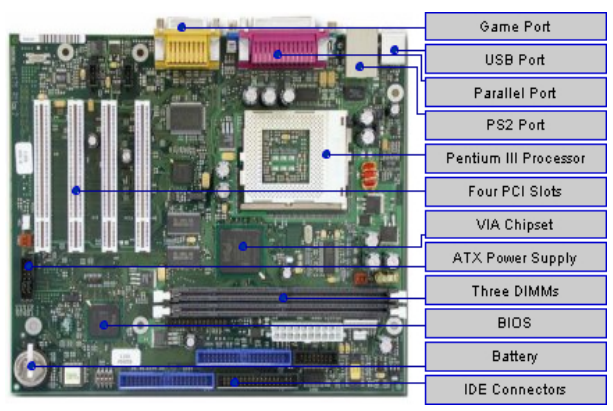

Fig. 3. A technical drawing of a motherboard

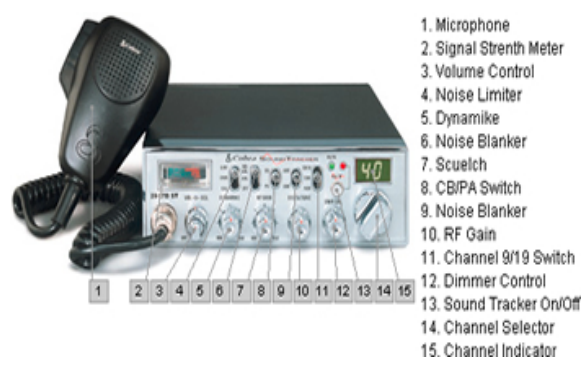

Fig. 2. A technical drawing of a cb radio

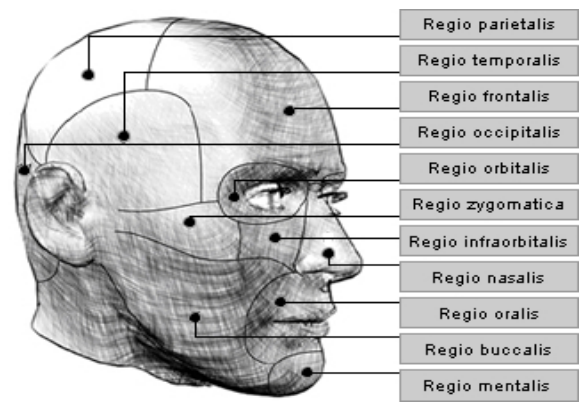

Fig. 4. A medical map of the regions of a human head

BLer can also be instructed to use a legend (see Figure 21), which is useful in cases where the labels (due to their size and number) do not all fit on the boundary of the enclosing rectangle. When a legend is used, labels of appropriate uniform size are used, each containing a number which refers to a particular line of the legend. The legend is treated as a floating object and can be manually placed anywhere around the resulting labelling.

As expected from a labelling tool, it supports storing, reloading and postprocessing of labellings. It provides advanced graphic functionality, including popup menus, printing capabilities, custom-zoom, fit-in window, selection, dragging and resizing of objects.

Figures 2, 3] and 4 depict some characteristic output examples of BLer.

\section{Reference}

1. M. Bekos, M. Kaufmann, A. Symvonis, and A. Wolff. Boundary labeling: Models and efficient algorithms for rectangular maps. In Janos Pach, editor, Proc. 12th Int. Symposium on Graph Drawing (GD'04), LNCS 3383, pp. 49-59, 2004. 